\title{
Article \\ Cognitive Reserve in Early Manifest Huntington Disease Patients: Leisure Time Is Associated with Lower Cognitive and Functional Impairment
}

\author{
Simone Migliore ${ }^{1}{ }^{\mathbb{D}}$, Giulia $D^{\prime}$ Aurizio $^{2}$, Eugenia Scaricamazza ${ }^{1} \mathbb{D}$, Sabrina Maffi ${ }^{1}$, Consuelo Ceccarelli ${ }^{3}$, \\ Giovanni Ristori ${ }^{4}$, Silvia Romano ${ }^{4}\left(\mathbb{D}\right.$, Anna Castaldo ${ }^{5}\left(\mathbb{D}\right.$, Mario Fichera ${ }^{5}$, , Giuseppe Curcio $^{2}$ (D) \\ and Ferdinando Squitieri ${ }^{1, * \mathbb{D}}$
}

Citation: Migliore, S.; D'Aurizio, G.; Scaricamazza, E.; Maffi, S.; Ceccarelli, C.; Ristori, G.; Romano, S.; Castaldo,

A.; Fichera, M.; Curcio, G.; et al. Cognitive Reserve in Early Manifest Huntington Disease Patients: Leisure Time Is Associated with Lower Cognitive and Functional Impairment. J. Pers. Med. 2022, 12, 36. https://doi.org/10.3390/jpm 12010036

Academic Editor: Valentina Bessi

Received: 3 December 2021

Accepted: 30 December 2021

Published: 3 January 2022

Publisher's Note: MDPI stays neutral with regard to jurisdictional claims in published maps and institutional affiliations.

Copyright: (C) 2022 by the authors. Licensee MDPI, Basel, Switzerland. This article is an open access article distributed under the terms and conditions of the Creative Commons Attribution (CC BY) license (https:// creativecommons.org/licenses/by/ $4.0 /)$.
1 Huntington and Rare Diseases Unit, Fondazione IRCCS Casa Sollievo Della Sofferenza Hospital, 71013 San Giovanni Rotondo, Italy; sim.migliore@gmail.com (S.M.); eugenia138@hotmail.com (E.S.); sabrina.maffi@gmail.com (S.M.)

2 Department of Biotechnological and Applied Clinical Sciences, University of L'Aquila, 67100 L'Aquila, Italy; giulia.daurizio@gmail.com (G.D.); giuseppe.curcio@univaq.it (G.C.)

3 Italian League for Research on Huntington and Related Diseases Foundation, 00185 Rome, Italy; consuelo.ceccarelli@lirh.it

4 Centre for Experimental Neurological Therapies, Department of Neuroscience, Mental Health and Sensory Organs, Faculty of Medicine and Psychology, S. Andrea Hospital, Sapienza University, 00189 Rome, Italy; giovanni.ristori@uniroma.it (G.R.); silvia.romano@uniroma1.it (S.R.)

5 Department of Medical Genetics and Neurogenetics, Fondazione IRCCS Istituto Neurologico Carlo Besta, 20133 Milan, Italy; anna.Castaldo@istituto-besta.it (A.C.); mario.fichera@istituto-besta.it (M.F.)

* Correspondence: ferdinandosquitieri@yahoo.it or f.squitieri@css-mendel.it

\begin{abstract}
We focused on Cognitive Reserve (CR) in patients with early Huntington Disease (HD) and investigated whether clinical outcomes might be influenced by lifetime intellectual enrichment over time. CR was evaluated by means of the Cognitive Reserve Index questionnaire (CRIq), an internationally validated scale which includes three sections: education, working activity, and leisure time. The clinical HD variables were quantified at three different time points (baseline-t0, 1 year follow up-t1 and 2 years follow up-t2) as per the Unified Huntington's Disease Rating Scale (UHDRS), an internationally standardized and validated scale including motor, cognitive, functional and behavioral assays. Our sample consisted of 75 early manifest patients, withclinical stage scored according to the Total Functional Capacity (TFC) scale. Our correlational analysis highlighted a significant inverse association between CRIq leisure time (CRIq_LA) and longitudinal functional impairment (namely, the differential TFC score between $\mathrm{t} 2$ and $\mathrm{t} 0$ or $\Delta \mathrm{TFC})(p<0.05)$, and the multidimensional progression of HD as measured by the composite UHDRS (cUHDRS, $p<0.01$ ). CRIq_LA was significantly and positively associated with better cognitive performances at all time points $(p<0.05)$. Our results suggest that higher is the CRIq_LA, milder is the progression of HD in terms of functional, multidimensional and cognitive outcome.
\end{abstract}

Keywords: cognitive engagement; neurodegenerative; executive functions; cognition

\section{Introduction}

Huntington disease (HD) is a hereditary neurodegenerative disease manifesting with neurological and mental symptoms which may begin at any age, mainly in adulthood. The gene mutation is a CAG triplet expansion that is detectable by a molecular test during the presymptomatic lifetime [1]. Usually, the genetic test is associated with specific psychological and genetic counselling [2]. Neurological features include movement disorders, among which chorea is the landmark, and progressive cognitive decline starting with early emotion recognition deficit [3], spatial memory problems [4], impaired attention functions [5], and finally overt dementia in the later stage of the disease. 
According to the Cognitive Reserve (CR) hypothesis, clinical expression of brain diseases might be attenuated among individuals with higher education or active lifestyles [6] People with greater intellectual enrichment are able to withstand more severe neuropathology before developing cognitive impairment or dementia. Two models of reserve have been described so far: the passive, or brain reserve model and the active, or CR model. The former hypothesizes that each individual disposes of a certain amount of brain capacity (determined by brain volume, number of neurons, etc.) to withstand the development of neurologic diseases [4]. In contrast, the latter speculates that the nervous system might actively cope with brain damage by using more efficient pre-existing neural networks or recruiting additional brain regions [6-8]. The passive and active models of CR can be modulated by psychosocial factors. Intellectual enrichment is generally evaluated in terms of educational attainment, vocabulary knowledge, and occupation level $[9,10]$, and many studies have shown that premorbid engagement in leisure activities might increase $C R$ and mitigate longitudinal cognitive impairment [11,12].

The CR hypothesis has been well-supported by studies involving various neurological conditions, such as Alzheimer's disease (AD) [12], Parkinson's disease (PD) [13], Multiple Sclerosis (MS) [14], stroke [15], traumatic brain injury [16], and age-related white matter ischemic changes [17].

On the contrary, little is known about $\mathrm{CR}$ in HD. While research has begun to disclose the biological factors impacting HD development [18], the effect of environmental factors on the onset, progression and severity of the disease is far less clear. Studies in animal models of HD hint that enriched environments may delay motor onset [19] and significantly affect cognitive impairment [20,21]. Moreover, studies on HD patients suggest that an active lifestyle and increased educational level have a beneficial effect on both clinical symptoms and brain activation in HD [22-26]. Specifically, HD subjects with higher cognitive reserve showed (a) slower decline in specific cognitive domains such as cognitive flexibility, working memory and inhibitory control; (b) slower volume loss in specific brain regions (i.e., putamen, left precuneus and the bilateral caudate), and (c) reinforced connectivity in subcortical and cortical brain structures (i.e., anterior cingulate cortex and left angular gyrus) [22-24].

Altogether, these data support the hypothesis that neuroplasticity is an intrinsic property of the living brain that enables it to reorganize its structure, function and networks in response to internal/external changes (i.e., brain pathologies or physical exercise) and may represent a potential protective factor in neurodegeneration [27]. Regional brain overactivity, which might represent a compensatory process in response to neurodegeneration [18,28], has been observed in both the presymptomatic and early stages of HD.

Our study aims to evaluate CR in patients with early manifest HD by investigating whether lifetime intellectual enrichment may be beneficial to cognitive and clinical performance. For this purpose, we used an international validated questionnaire, the Cognitive Reserve Index questionnaire (CRIq), which assesses the acquired lifetime CR in three different areas: education, working activities, and leisure time activities.

\section{Materials and Methods}

\subsection{Subjects}

Starting from a sample of 177 early manifesting patients with at least three yearly clinical examinations, we selected 75 patients based on the following inclusion criteria: (1) motor age at onset above 20 years; (2) Unified Huntington Disease Rating Scale (UHDRS) - Total Motor Score (TMS) > 10 and Diagnostic Confidence Level (DCL) equal to 4 at baseline; (3) UHDRS Total Function Capacity (TFC) $>6$ at baseline; (4) no history of neurological conditions other than HD, substance dependence, or developmental disorder affecting cognition at baseline assessment; and (5) no severe psychiatric manifestations. A full patient description is reported in Table 1 . HD was genetically confirmed in all cases, with gene expansions being greater than $\geq 40$ CAG repeats. This cohort was recruited by three ENROLL-HD Centres in Italy, e.g., the LIRH Foundation (the coordinator site, Rome), 
IRCCS Istituto Neurologico Carlo Besta (Milan); and Sant' Andrea University Hospital (Rome). ENROLL-HD is a large worldwide research platform for observational studies on HD. HD family participants are assessed yearly by standardized medical, motor, behavioral, functional and cognitive measures [29]. All raters are required to be annually certified before performing ENROLL-HD clinical assays.

Table 1. Clinical and demographic characteristics of the study sample.

\begin{tabular}{cc}
\hline \multicolumn{2}{c}{ Early Manifest HD Cohort } \\
\hline Sample Number & 75 \\
\hline Male-Female & $47-28$ \\
\hline Age at baseline & $47.2 \pm 12.5$ \\
(range) & $(27-78)$ \\
\hline Education level in years & $11.65 \pm 4.6$ \\
(range) & $(8-18)$ \\
\hline Age at motor onset & $47.5 \pm 12.3$ \\
(range) & $(22-70)$ \\
\hline CAG repeat number & $43.7 \pm 2.3$ \\
(range) & $(40-49)$ \\
\hline
\end{tabular}

Our study conforms with the World Medical Association Declaration of Helsinki. It received approval by the Institutional Review Board of the coordinator site (LIRH Foundation, prot. number 102/14, approved in date 28 May 2014); all participants signed a declaration of informed consent.

\subsection{Clinical Measures}

All patients were assessed by health professionals with expertise in HD in accordance with ENROLL-HD guidelines [29]. Evaluations were performed at baseline (time 0; t0) and repeated at 1-year (time 1; t1) and 2-year (time 2; t2) follow-ups by means of the UHDRS [30], which includes motor, cognitive and functional domains. The TMS is a standardized and validated tool for rating motor impairment consisting of 31 items; each item can be scored from 0 (indicating normal performance) to 4 (indicating the most severe impairment) [30]. The TFC is a standardized and validated HD assay of overall functional capacity considering the following domains: occupation, financial management, domestic chores, and daily life activities, with a total score ranging from 13 (normal function) to 0 (complete loss of function) [30,31].

We performed a comprehensive cognitive evaluation including: (1) Categorical Verbal Fluency Test (VFT); (2) Stroop Color Reading Test (SCR); (3) Stroop Word Reading (SWR); (4) Symbol Digit Modalities Test (SDMT) in the written response format; and (5) Mini Mental State Examination (MMSE), according to ENROLL-HD protocol. We calculated the composite UHDRS (cUHDRS), a multidimensional measure of motor (TMS), cognitive (SDMT and SWR) and functional (TFC) outcome, recently proposed as a sensitive tool for monitoring clinical progression in early HD [32].

\subsection{Cognitive Reserve}

CR was assessed by means of the CRIq, an internationally validated instrument that is currently available in ten different languages. The questionnaire is composed of twenty items grouped into three section subscores: (1) years of formal and informal education (CRIq_Edu); (2) working activity experience according to degree of responsibility and cognitive demands (CRIq_WA), with five different levels of working activities available dealing with the degree of intellectual involvement and personal responsibility; and (3) leisure activity, calculated based on the frequency of several activities such as reading, housekeeping, driving, hobbies, travelling etc., on a weekly, monthly, yearly, or stable basis (CRIq_LA) [33]. 
The total score (CRIq_Tot) and the three subscores (CRIq_Edu, CRIq_WA, CRIq_LA) were considered in our analysis. Experienced health professionals conducted semi-structured interviews to gather pertinent information. The CRIq instructions and the Excel file for automatic calculation of subscores are available at https://dpg.unipd.it/en/criq (accessed on 15 January 2019). CRIq is an efficient and reliable tool for measuring CR [33]; specifically, it is short and easy to administer and thus easily to be included in standard assessments.

\subsection{Statistical Analysis}

First, we conducted an exploratory Pearson's correlation analysis in order to detect potential relationships between the CRIq scores (CRIq_Tot, CRIq_Edu, CRIq_WA, CRIq_LA) and the clinical and genetic variables under investigation (Age at Onset (AO), expanded CAG repeats, $\triangle \mathrm{TMS}$ (the difference between the TMS score at $\mathrm{t} 2$ and $\mathrm{t} 0$ ), $\triangle \mathrm{TFC}, \mathrm{cUHDRS}$, cognitive variables (Categorical Verbal Fluency Test, correct response; Stroop Color Reading, correct responses; Stroop Word Reading, correct responses; Symbol Digit Modalities Test, correct responses), and Mini Mental State Examination). The correlation analysis was performed for all time points ( $\mathrm{t} 0, \mathrm{t} 1$, and $\mathrm{t} 2$ ). In addition, we investigated the correlation between the mutation size (i.e., expanded CAG repeat number) with each clinical variable.

Patients were then split into two groups depending on whether the CRIq scores obtained were normal or impaired. CRIq subscores were considered pathological when below 1 Standard Deviation (SD) from the control mean; for more details, see [33].

All dependent variables were submitted into a one-way ANOVA comparing the performance of patients with normal and impaired CRIq subscores.

Alpha level was conventionally fixed to $\leq 0.05$. All statistical analyses were performed using IBM SPSS Statistics for Macintosh, version 25.0 (IBM Corp., Armonk, NY, USA).

\section{Results}

\subsection{Patients' Characteristics}

Our cohort $(\mathrm{n}=75)$ included 28 females and 47 males. The demographic characteristics of the sample included age at baseline of $47.2 \pm 12.5$ years (range 27-78 years), education level of $11.65 \pm 4.6$ years (range 8-18 years), age at motor onset $47.5 \pm 12.3$ years (range 22-70 years), and expanded CAG repeat number of $43.7 \pm 2.3$ (range 40-49 CAG repeats). The clinical and demographic characteristics are summarized in Table 1. Genetic characteristics with mutation size, including the expanded CAG repeat number, are reported in Table S1.

\subsection{Correlation between $C R$, Clinical and Cognitive Scores}

The preliminary Pearson's analysis highlighted various significant associations between CRIq scores, in particular the CRI_LA subscore, and the clinical/cognitive variables (Figures 1 and 2). Specifically, among the clinical variables, significant positive correlations were found between CRIq_LA and $\triangle \mathrm{TFC}(p=0.037 ; r=0.242)$, cUHDRS_t0 $(p=0.002$; $r=0.349)$, cUHDRS_t1 $(p=0.004 ; r=0.332)$ and cUHDRS_t2 $(p=0.003 ; r=0.344)$ (Figure 1 , Table 2). 


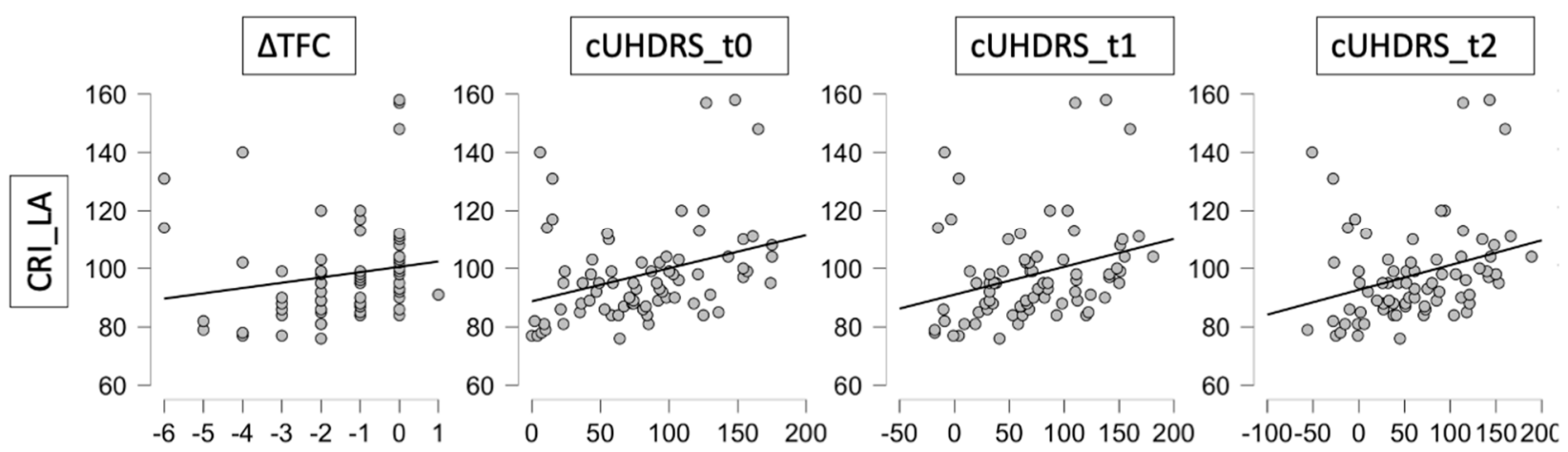

Figure 1. Correlation Matrix plots between clinical variables and Cognitive Reserve Index Leisure Activities.

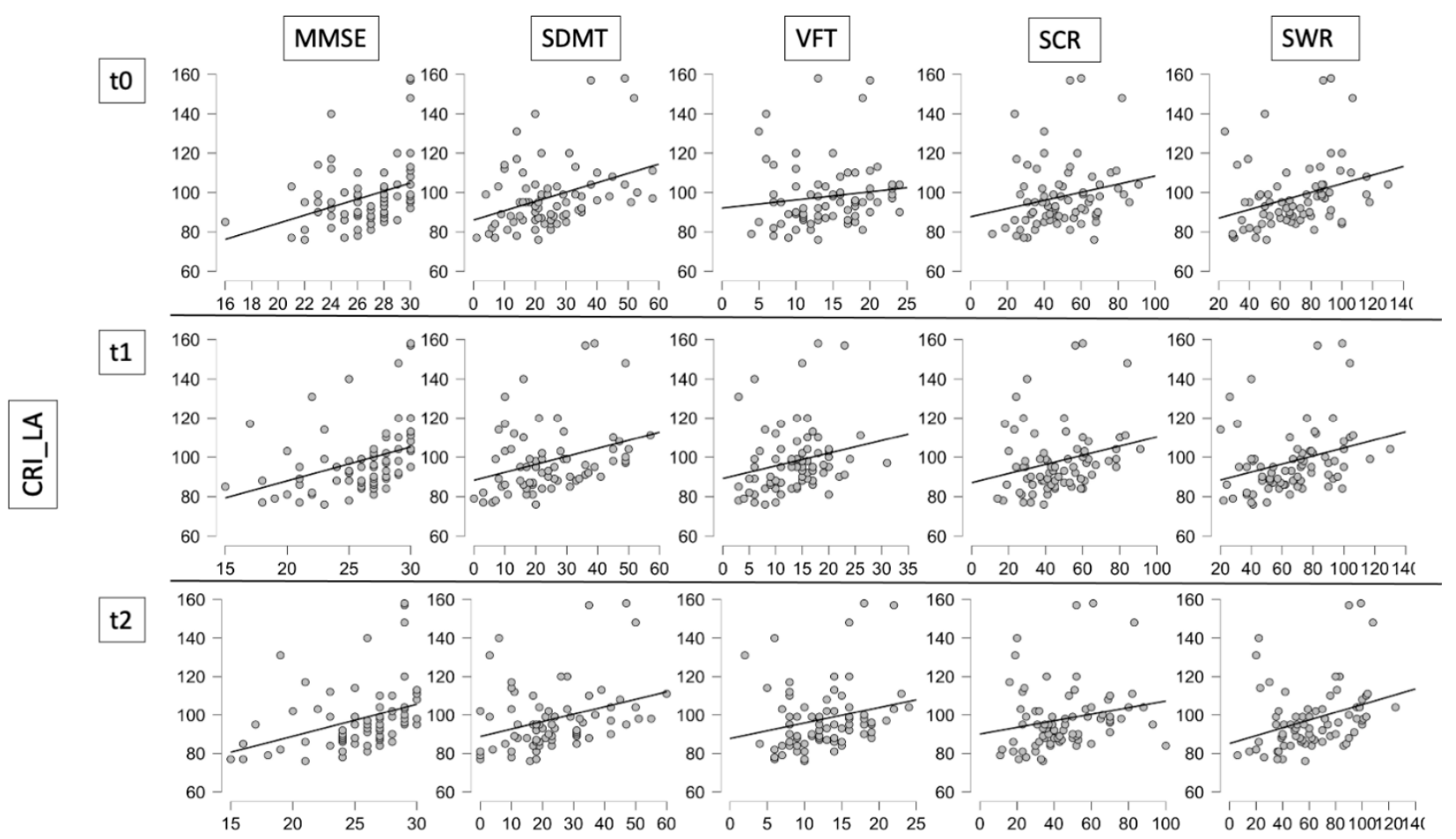

Figure 2. Correlation Matrix plots between cognitive variables and Cognitive Reserve Index Leisure Activities.

Table 2. Pearson's $r$ (and related level of significance) between Cognitive Reserve Index subscores and clinical variables.

\begin{tabular}{|c|c|c|c|c|c|c|c|c|c|c|c|c|}
\hline & \multicolumn{2}{|c|}{ Age of Onset } & \multicolumn{2}{|c|}{$\Delta \mathrm{TFC}$} & \multicolumn{2}{|c|}{$\Delta \mathrm{TMS}$} & \multicolumn{2}{|c|}{ cUHDRS_t0 } & \multicolumn{2}{|c|}{ cUHDRS_t1 } & \multicolumn{2}{|c|}{ cUHDRS_t2 } \\
\hline & $r$ & $p$ & $r$ & $p$ & $r$ & $p$ & $r$ & $p$ & $r$ & $p$ & $r$ & $p$ \\
\hline CRIq_Edu & 0.156 & 0.181 & 0.036 & 0.758 & -0.104 & 0.375 & 0.049 & 0.690 & 0.029 & 0.805 & 0.018 & 0.878 \\
\hline CRIq_WA & 0.026 & 0.824 & 0.058 & 0.619 & -0.141 & 0.228 & 0.058 & 0.624 & 0.078 & 0.508 & 0.065 & 0.580 \\
\hline CRIq_LA & 0.095 & 0.418 & 0.242 & 0.037 & -0.150 & 0.200 & 0.349 & 0.002 & 0.332 & 0.004 & 0.344 & 0.003 \\
\hline CRIq_Tot & 0.170 & 0.146 & 0.117 & 0.316 & -0.117 & 0.319 & 0.191 & 0.100 & 0.179 & 0.124 & 0.149 & 0.203 \\
\hline
\end{tabular}

With regard to the cognitive variables, at baseline the analysis showed significant positive correlations between CRIq_LA and SDMT $((p=0.001 ; r=0.392)$, VFT $(p=0.036$, $r=0.240), \operatorname{SCR}(p=0.015, r=0.280), \operatorname{SWR}(p=0.002, r=0.348)$ and MMSE $(p=0.003$, $r=0.340$ ) (see Figure 2 and Table $3(\mathrm{a})$ ). We found a significant positive correlation between 
CRIq_Tot and SDMT $(p<0.039 ; r=0.239)$ and MMSE $(p=0.012, r=0.294)$ as well (see Table $3(a))$.

Table 3. Pearson's $r$ (and related level of significance) between Cognitive Reserve Index subscores and cognitive variables: (a) Pearson's $r$ (and related level of significance) between Cognitive Reserve Index subscores and MMSE, SDMT, VTF, SCR and SWR at baseline (t0); (b) Pearson's $r$ (and related level of significance) between Cognitive Reserve Index subscores and MMSE, SDMT, VTF, SCR and SWR at 1 year follow-up (t1); (c) Pearson's $r$ (and related level of significance) between Cognitive Reserve Index subscores and MMSE, SDMT, VTF, SCR and SWR at two-year follow-up (t2).

\begin{tabular}{|c|c|c|c|c|c|c|c|c|c|c|}
\hline \multicolumn{11}{|c|}{ (a) } \\
\hline & \multicolumn{2}{|c|}{ MMSE } & \multicolumn{2}{|c|}{ SDMT } & \multicolumn{2}{|c|}{ VFT } & \multicolumn{2}{|c|}{ SCR } & \multicolumn{2}{|c|}{ SWR } \\
\hline & $r$ & $p$ & $r$ & $p$ & $r$ & $p$ & $r$ & $p$ & $r$ & $p$ \\
\hline CRIq_Edu & 0.206 & 0.082 & 0.026 & 0.825 & 0.080 & 0.494 & 0.019 & 0.870 & 0.117 & 0.319 \\
\hline CRIq_WA & 0.146 & 0.220 & 0.137 & 0.242 & -0.160 & 0.171 & -0.016 & 0.892 & 0.079 & 0.500 \\
\hline CRIq_LA & 0.340 & 0.003 & 0.392 & 0.001 & 0.240 & 0.036 & 0.280 & 0.015 & 0.348 & 0.002 \\
\hline CRIq_Tot & 0.294 & 0.012 & 0.239 & 0.039 & 0.025 & 0.831 & 0.100 & 0.393 & 0.220 & 0.058 \\
\hline \multicolumn{11}{|c|}{ (b) } \\
\hline & \multicolumn{2}{|c|}{ MMSE } & \multicolumn{2}{|c|}{ SDMT } & \multicolumn{2}{|c|}{ VFT } & \multicolumn{2}{|c|}{ SCR } & \multicolumn{2}{|c|}{ SWR } \\
\hline & $r$ & $p$ & $r$ & $p$ & $r$ & $p$ & $r$ & $p$ & $r$ & $p$ \\
\hline CRIq_Edu & 0.205 & 0.083 & -0.005 & 0.970 & 0.106 & 0.365 & 0.071 & 0.547 & 0.075 & 0.522 \\
\hline CRIq_WA & 0.192 & 0.098 & 0.114 & 0.337 & 0.027 & 0.820 & 0.064 & 0.588 & 0.082 & 0.485 \\
\hline CRIq_LA & 0.396 & $<0.001$ & 0.359 & 0.002 & 0.265 & 0.022 & 0.294 & 0.010 & 0.330 & 0.004 \\
\hline CRIq_Tot & 0.337 & 0.003 & 0.192 & 0.103 & 0.156 & 0.183 & 0.171 & 0.142 & 0.200 & 0.085 \\
\hline \multicolumn{11}{|c|}{ (c) } \\
\hline & \multicolumn{2}{|c|}{ MMSE } & \multicolumn{2}{|c|}{ SDMT } & \multicolumn{2}{|c|}{ VFT } & \multicolumn{2}{|c|}{ SCR } & \multicolumn{2}{|c|}{ SWR } \\
\hline & $r$ & $p$ & $r$ & $p$ & $r$ & $p$ & $r$ & $p$ & $r$ & $p$ \\
\hline CRIq_Edu & 0.193 & 0.100 & -0.021 & 0.860 & 0.082 & 0.486 & -0.001 & 0.991 & 0.046 & 0.694 \\
\hline CRIq_WA & 0.205 & 0.080 & 0.087 & 0.456 & 0.069 & 0.554 & 0.037 & 0.750 & 0.079 & 0.499 \\
\hline CRIq_LA & 0.404 & $<0.001$ & 0.354 & 0.002 & 0.252 & 0.029 & 0.253 & 0.028 & 0.374 & 0.001 \\
\hline CRIq_Tot & 0.326 & 0.005 & 0.178 & 0.128 & 0.167 & 0.153 & 0.113 & 0.334 & 0.200 & 0.085 \\
\hline
\end{tabular}

At $\mathrm{t} 1$, the analysis showed significant positive correlations between CRIq_LA and $\operatorname{SDMT}(p=0.002) ; r=0.359)$, VFT $(p=0.022, r=0.265)$, SCR $(p=0.01, r=-0.294)$, SWR $(p=0.004, r=0.330)$ and MMSE $(p<0.001, r=0.396$; Figure 2 and Table 3(b) $)$ scores. We found a significant positive correlation between CRIq_Tot and MMSE $(p=0.003, r=0.337)$ as well (see Table $3(b)$ ).

Finally, at $\mathrm{t} 2$ the analysis showed significant positive correlations between CRIq_LA and SDMT $(p=0.002 ; r=0.354)$, VFT $(p=0.029, r=0.252)$, SCR $(p=0.028, r=0.253)$, SWR $(p=0.001, r=0.374)$ and MMSE $(p<0.001, r=0.404)$ (Figure 2 and Table $3(\mathrm{c}))$. We again found a significant positive correlation between CRIq_Tot and MMSE ( $p=0.005, r=0.326$; see Table 3 (c)).

The expanded CAG repeat number showed a significant negative correlation with CRIq_Edu $(p=0.002, r=-0.348)$, CRIq_WA $(p=0.005, r=-0.320)$ and CRIq_Tot $(p=0.001$, $r=-0.372$; Table S2). The mutation size did not affect the relationship of CRIq_LA (Table S2) with clinical ( $\triangle \mathrm{TMS}, \triangle \mathrm{TFC}$, cUHDRS; Table S2) or cognitive variables (SDMT, VFT, SCR, SWR and MMSE; Table S3).

\subsection{Comparison between Groups with Impaired and Normal Cognitive Reserve Indices}

Patients were split into two groups depending on the totalization of a normal or impaired score at CRI assessment; hence a one-way ANOVA comparison was performed.

CRIq Leisure Activity: Among the clinical variables, we found a significant Group effect $\left(\mathrm{F}_{1,73}=5.94 ; p=0.017\right)$ with regard to the $\triangle \mathrm{TFC}$, which resulted higher in the low 
CRIq_LA group $(-2.08 \pm 0.28)$ in comparison to the high CRIq_LA group $(-1.2 \pm 0.21)$. Moreover, the one-way ANOVA showed a significant group effect on cUHDRS at t0 $\left(\mathrm{F}_{1,73}=11.29 ; p=0.001\right), \mathrm{t} 1\left(\mathrm{~F}_{1,73}=13.87 ; p=0.0001\right)$ and $\mathrm{t} 2\left(\mathrm{~F}_{1,73}=11.16 ; p=0.0001\right)$, indicating a more pronounced multidimensional progression in the low CRIq_LA than in the high CRIq_LA group (t0: $54.34 \pm 8.23$ vs. $92.28 \pm 6.69)(\mathrm{t} 1: 41.09 \pm 8.41$ vs. $85.48 \pm 7.14$; t2: $30.09 \pm 9.64$ vs. $75.5 \pm 8.13$ ). No significant differences emerged for $\triangle$ TMS (see Table 4 for further details).

Table 4. Clinical and cognitive scores in impaired and normal cognitive reserve leisure activities groups.

\begin{tabular}{|c|c|c|c|c|}
\hline & & $\begin{array}{c}\text { CRI_LA Impaired Group } \\
\qquad(n=24) \\
\text { Mean } \pm \text { SE }\end{array}$ & $\begin{array}{l}\text { CRI_LA Normal Group } \\
\qquad(n=51) \\
\text { Mean } \pm \text { SE }\end{array}$ & $p$ \\
\hline \multirow{5}{*}{ Clinical variables } & $\Delta \_\mathrm{TFC}$ & $-2.08 \pm 0.29$ & $-1.2 \pm 0.21$ & 0.017 \\
\hline & $\Delta \_\mathrm{TMS}$ & $9.79 \pm 1.94$ & $8.25 \pm 1.24$ & 0.496 \\
\hline & cUHDRS_t0 & $54.34 \pm 8.23$ & $92.28 \pm 6.69$ & 0.001 \\
\hline & cUHDRS_t1 & $41.09 \pm 8.41$ & $85.48 \pm 7.14$ & $<0.001$ \\
\hline & cUHDRS_t2 & $30.09 \pm 9.64$ & $75.50 \pm 8.13$ & $<0.001$ \\
\hline \multirow{3}{*}{ Cognitive variables } & MMSE & $25.34 \pm 0.60$ & $26.98 \pm 0.37$ & 0.019 \\
\hline & SDMT & $17.54 \pm 1.70$ & $28.58 \pm 1.92$ & 0.001 \\
\hline & VFT & $11.87 \pm 0.84$ & $14.94 \pm 0.75$ & 0.016 \\
\hline \multirow[t]{2}{*}{ Baseline } & SCR & $41.41 \pm 3.19$ & $52.88 \pm 2.42$ & 0.007 \\
\hline & SWR & $58.75 \pm 4.39$ & $75.02 \pm 3.35$ & 0.006 \\
\hline \multirow{5}{*}{$\begin{array}{c}\text { Cognitive variables } \\
- \\
1 \text { year } \\
\text { follow-up }\end{array}$} & MMSE & $23.62 \pm 0.74$ & $26.78 \pm 0.41$ & $<0.001$ \\
\hline & SDMT & $16.04 \pm 1.84$ & $28.06 \pm 1.91$ & $<0.001$ \\
\hline & VFT & $10.7 \pm 1$ & $14.94 \pm 0.8$ & 0.003 \\
\hline & SCR & $39.25 \pm 2.71$ & $50.17 \pm 2.63$ & 0.013 \\
\hline & SWR & $54.16 \pm 3.92$ & $72.6 \pm 3.49$ & 0.002 \\
\hline \multirow{5}{*}{$\begin{array}{c}\text { Cognitive variables } \\
- \\
2 \text { years } \\
\text { follow-up }\end{array}$} & MMSE & $23.08 \pm 0.8$ & $26.56 \pm 0.41$ & $<0.001$ \\
\hline & SDMT & $15.5 \pm 1.81$ & $27.12 \pm 2.02$ & 0.001 \\
\hline & VFT & $10.29 \pm 0.74$ & $13.58 \pm 0.69$ & 0.005 \\
\hline & SCR & $36.75 \pm 3.81$ & $49.17 \pm 2.78$ & 0.012 \\
\hline & SWR & $48.42 \pm 4.58$ & $69.16 \pm 3.73$ & 0.002 \\
\hline
\end{tabular}

Regarding cognitive variables, a significant group effect was observed, showing globally poorer performances in the group with low CRIq_LA compared to the high CRIq_LA group at baseline. The detailed statistical results are listed as follows: MMSE $\left(\mathrm{F}_{1,70}=5.74 ; p=0.019 ; 25.34 \pm 0.60\right.$ vs. $\left.26.98 \pm 0.37\right), \operatorname{SDMT}\left(\mathrm{F}_{1,73}=13.2 ; p=0.001 ;\right.$ $17.54 \pm 1.70$ vs. $28.58 \pm 1.92)$, VFT $\left(\mathrm{F}_{1,73}=6.03 ; p=0.016 ; 11.87 \pm 0.84\right.$ vs $\left.14.94 \pm 0.75\right)$, $\operatorname{SCR}\left(\mathrm{F}_{1,73}=7.61 ; p=0.007 ; 41.41 \pm 3.19\right.$ vs. $\left.52.88 \pm 2.42\right)$ and $\operatorname{SWR}\left(\mathrm{F}_{1,73}=8.02 ; p=0.006\right.$; $58.75 \pm 4.39$ vs. $75.02 \pm 3.35$; see Table 4 for further details).

The one-way ANOVA confirmed a significant Group effect on MMSE $\left(\mathrm{F}_{1,73}=16.1\right.$; $p=0.0001 ; 23.62 \pm 0.74$ vs. $26.78 \pm 0.41)$, $\operatorname{SDMT}\left(\mathrm{F}_{1,71}=15.1 ; p=0.0001 ; 16.04 \pm 1.84\right.$ vs. $28.06 \pm 1.91)$, VFT $\left(\mathrm{F}_{1,73}=9.72 ; p=0.003 ; 10.7 \pm 1\right.$ vs. $\left.14.94 \pm 0.8\right)$, $\mathrm{SCR}\left(\mathrm{F}_{1,73}=6.54 ;\right.$ $p=0.013)(39.25 \pm 2.71$ vs. $50.17 \pm 2.63)$ and SWR $\left(\mathrm{F}_{1,73}=10.22 ; p=0.002 ; 54.16 \pm 3.92\right.$ vs. $72.6 \pm 3.49$ ) at 1 year follow-up, indicating poorer performance in the impaired CRIq_LA group with respect to the normal CRIq_LA group (see Table 4 for further details).

The one-way ANOVA revealed a significant group effect on MMSE $\left(\mathrm{F}_{1,72}=17.8\right.$; $p=0.0001 ; 23.08 \pm 0.8$ vs. $26.56 \pm 0.41)$, $\operatorname{SDMT}\left(\mathrm{F}_{1,73}=13.1 ; p=0.001 ; 15.5 \pm 1.81\right.$ vs $27.12 \pm 2.02)$, VFT $\left(\mathrm{F}_{1,73}=8.36 ; p=0.005 ; 10.29 \pm 0.74\right.$ vs. $\left.13.58 \pm 0.69\right), \operatorname{SCR}\left(\mathrm{F}_{1,73}=6.63 ;\right.$ $p=0.012 ; 36.75 \pm 3.81$ vs. $49.17 \pm 2.78)$ and $\operatorname{SWR}\left(\mathrm{F}_{1,73}=10.87 ; p=0.002 ; 48.42 \pm 4.58 \mathrm{vs}\right.$ $69.16 \pm 3.73$ ) at 2 years follow-up, showing poorer performance in the group with low CRIq_LA with respect to high CRI_LA (see Table 4 for further details).

CRIq Total: No statistically significant differences between groups were observed in any of the clinical and cognitive variables (see Table S4.1 in Supplementary Materials). 
CRIq Education: No statistically significant differences between groups were observed in any of the clinical and cognitive variables (see Table S4.2 in Supplementary Materials).

CRIq Working activity: No statistically significant differences between groups were observed in any of the clinical and cognitive variables considered (see Table S4.3 in Supplementary Materials).

CRIq_Edu: Cognitive Reserve Index education; CRIq_WA: Cognitive Reserve Index Working Activities; CRIq_LA: Cognitive Reserve Index Leisure Activities; CRIq_TOT: Cognitive Reserve Index Total Score.

$\triangle T F C$ : the difference between the Total Functional Capacity score at $\mathrm{t} 2$ and $\mathrm{t} 0 ; \Delta \mathrm{TMS}$ : the difference between the Total Motor Score at $\mathrm{t} 2$ and $\mathrm{t} 0$.

cUHDRS_t0: composite Unified Huntington's Disease Rating Scale at baseline; cUHDRS_t1: composite UHDRS at 1-year follow-up; cUHDRS_t2: composite UHDRS at 2-year follow-up.

CRIq_Edu: Cognitive Reserve Index education; CRIq_WA: Cognitive Reserve Index Working Activities; CRIq_LA: Cognitive Reserve Index Leisure Activities; CRIq_Tot: Cognitive Reserve Index Total Score.

MMSE: Mini Mental State Examination; SDMT: Symbol Digit Modality Test; VFT: Categorical Verbal Fluency Test; SCR: Stroop Color Reading Test; SWR: Stroop Word Reading Test.

CRIq_LA: Cognitive Reserve Index Leisure Activities; $\triangle T F C$ : the difference between the Total Functional Capacity score at $\mathrm{t} 2$ and $\mathrm{t} 0 ; \Delta \mathrm{TMS}$ : the difference between the Total Motor Score at $\mathrm{t} 2$ and $\mathrm{t} 0$.

cUHDRS_t0: composite Unified Huntington's Disease Rating Scale at baseline; cUHDRS_t1: composite UHDRS at 1-year follow-up; cUHDRS_t2: composite UHDRS at 2-year follow-up.

MMSE: Mini Mental State Examination; SDMT: Symbol Digit Modality Test; VFT: Categorical Verbal Fluency Test; SCR: Stroop Color Reading Test; SWR: Stroop Word Reading Test.

SE: standard error; NS: not significant; t0: baseline; $\mathrm{t} 1$ : 1 year follow-up; 2 : 2 years follow-up.

CRIq_LA: Cognitive Reserve Index Leisure Activities; $\triangle T F C$ : the difference between the Total Functional Capacity score at $\mathrm{t} 2$ and $\mathrm{t} 0 ; \Delta \mathrm{TMS}$ : the difference between the Total Motor Score at $\mathrm{t} 2$ and $\mathrm{t} 0$.

CRIq_LA: Cognitive Reserve Index Leisure Activities; MMSE: Mini Mental State Examination; SDMT: Symbol Digit Modality Test; VFT: Categorical Verbal Fluency Test; SCR: Stroop Color Reading Test; SWR: Stroop Word Reading Test; t0: baseline; t1: 1 year follow-up; t2: 2 years follow-up.

\section{Discussion}

The lifetime intellectual enrichment may significantly improve the cognitive and clinical performances in several neurological conditions [7]. Growing evidence is in favor of a positive influence of environment and cognition on the course of neurodegenerative diseases, including HD. For instance, bilingualism seems to exert a neuroprotective effect by influencing structural and metabolic brain changes [34]. Moreover, functional compensation is leveraged by lifestyle, i.e., the enhancement of physical and mental activities in AD and HD [34,35]. Additional examples concern the effect of educational level [25] and of biological factors $[18,36]$ on neural compensation and eventually on clinical decline in HD.

One general limitation of studies on CR in HD is either a limited sample size or difficulty in analyzing specific tasks [24]. However, in an attempt to overcome this limitation, in this study we used the CRIq, an international questionnaire composed of several subscales, which has been used in normal and elderly populations and in other pathological conditions [37]. Among its subscales, we found the leisure time score exerted a major significant effect on clinical outcomes. Interestingly, we found that a higher leisure time was correlated with lesser cognitive and functional HD decline over time in early manifest 
HD subjects. Our findings are in line with the documented evidence of the beneficial effect of leisure activities on cognitive decline in the early stages of non-HD neurodegenerative diseases [38]. Leisure activities, that is, the time when a person is not working and can relax and do things that they enjoy (i.e., reading, theater, television, driving, internet, gardening, music etc.) contribute to increment a specific cognitive reserve that modulates the negative effect of brain pathology on cognition and independence in daily life. On the contrary, no effect of leisure time was observed on clinical motor variables (i.e., TMS); this is not surprising, as the cognitive reserve is mainly developed by exercising mental capacity, which seems to influence mainly the cognitive and functional domains [39].

HD patients with a higher reserve are able to withstand a more severe neuropathological burden; this protective effect of $\mathrm{CR}$ is shown by greater cognitive functioning as well as by less impairment in daily living activities. We found a long-lasting beneficial effect of $C R$ on cognition and clinical outcomes that is consistent with previous $C R$ research in $A D$ and MS [40,41], and which could represent a potential neuroprotective target in HD as well.

We found that CRIq_Tot subscore, which includes education and work in addition to leisure activities, correlated with some cognitive measures. Specifically, CRIq_Tot correlated with MMSE over time, a screening measure of global cognitive impairment, as well as with SDMT at baseline. These results suggest a possible, although marginal influence of other $\mathrm{CR}$ variables on global cognitive deterioration over time.

Recent studies in HD show the existence of several neural mechanisms of compensation, suggesting the presence of a spread neural network to support cognitive dysfunctions; for a review, see [24,28]. High cognitive reserve seems to be associated with a reduced compensatory effect. Specifically, subjects who are in the early HD stages and experience an active cognitive lifestyle show reduced volume loss and reduced compensation in connectivity strength in specific brain regions related to executive control networks with respect to subjects with poorer cognitive lifestyle engagement [22,23]. These data are in accordance with the neural efficiency hypothesis [42]; people with a higher reserve require fewer cerebral resources to perform the same cognitive tasks than others with a lesser reserve.

Our study supports the hypothesis that at the beginning of the disease, leisure time plays a central role in preventing cognitive and functional degeneration over time when compared with education and work. A possible explanation is that the cognitive reserve provides constant exercise of the mental capacity during the whole life span. Conversely, it is possible that education may exercise this capacity only in the first part of life, while work may lose its power to influence this capacity over time as routines take over. After the formal education ends and work becomes more regular, leisurely cognitive activities provide ongoing mental exercise and stimulation that is critical for further development and maintenance of the cognitive reserve. Therefore, CR is not fixed over time, and it is modifiable in later life. This is supported by the evidence that cognitive stimulation and physical exercise increase neuroplasticity and promote neurogenesis [35]. The main biological mechanism underlying neural plasticity has been postulated to be an increase in BDNF levels. BDNF mediates the effect on brain engagement and cognitive reserve driven by physical exercise or by brain stimulation [43]. Modulating the cognitive reserve through lifestyle changes and/or through development of appropriate cognitive stimulation may represent a potential new avenue to counteract neurodysfunction and neurodegeneration.

Our findings highlight a negative correlation between mutation size and measured CR, thus supporting the interesting hypothesis that a larger expanded CAG repeat number in HTT gene is correlated with a lower patient CR. Even though our study did not reveal any mutation size amplification of the CRIq_LA effect on clinical measures, our data suggest a combinational environmental and genetic influence on the clinical development of the disease. The missing effect of the mutation on the relationship between CRIq_LA and the clinical measures may be explained by the relatively homogeneous CAG repeat number and by the limited range of expansions in our population. Larger studies including cohorts 
carrying higher repeat expansions (i.e., large mutations beyond 50 CAG repeats affecting young patients) may eventually highlight such a neurobiological effect of the mutation.

However, our study has limitations. First, even if we enrolled patients without severe psychiatric manifestations, we cannot fully exclude a possible influence of depression or anxiety on cognition. Second, the observational design of our study did not allow us to make causal inferences into the correlations between leisure time and the cognitive and functional domains.

Despite these limitations, our findings support the interesting hypothesis that in addition to possible genetic modifiers, the environment may consistently contribute to disease course modification and may represent a therapeutic target. Further studies on larger cohorts investigating the role of leisure time in HD are required in order to confirm our hypothesis and to address conclusions.

\section{Conclusions}

In conclusion, our results highlighting a combined effect of environmental and neurobiological factors (i.e., the expanded CAG repeat number in HTT gene) on the development of HD suggest that the CR may be affected in HD, and that increased leisure time lowers HD progression as measured by functional and multidimensional variables, with more preserved cognitive status over time. These aspects could be considered in clinical management to address HD by directing patients to specific cognitive training programs and clinical trial recruitments in order to minimize potential selection bias.

Supplementary Materials: The following are available online at https:/ /www.mdpi.com/article / 10.3390/jpm12010036/s1, Table S1: CAG expansion in our study sample; Table S2: Correlations between CAG expansions and clinical variables; Table S3: Correlations between CAG expansions and cognitive variables at three time points; Table S4.1: Clinical and cognitive scores in impaired and normal cognitive reserve total index groups; Table S4.2: Clinical and cognitive scores in impaired and normal cognitive reserve education groups; Table S4.3: Clinical and cognitive scores in impaired and normal cognitive reserve working activity index groups.

Author Contributions: Conceptualization, S.M. (Simone Migliore) and F.S.; Data curation, E.S., S.M. (Sabrina Maffi), C.C., G.R., S.R., A.C. and M.F.; Formal analysis, G.D. and G.C.; Investigation, E.S., S.M. (Sabrina Maffi), C.C., G.R., S.R., A.C. and M.F.; Methodology, S.M. (Simone Migliore) and F.S.; Writing-original draft, S.M. (Simone Migliore) and F.S. All authors have read and agreed to the published version of the manuscript.

Funding: This research received funds from the Italian Ministry of Health (RC2101MH09 and RF2016-02364123 to S.M. (Simone Migliore), S.M. (Sabrina Maffi), F.S.).

Institutional Review Board Statement: This study conforms with the World Medical Association Declaration of Helsinki. It received approval by the Institutional Review Board of the coordinator site (LIRH Foundation, prot. number 102/14, approved in date 28 May 2014).

Informed Consent Statement: Informed consent was obtained from all subjects involved in the study.

Data Availability Statement: The data that support the findings of this study are available from the corresponding author upon reasonable request.

Acknowledgments: We are grateful to all families and patients from the LIRH Foundation Network of Huntington disease patients' associations (LIRH-Puglia, LIRH-Tuscany, Noi Huntington) for their continuous support of LIRH research initiatives. LIRH Foundation received funding from the Cure Huntington's Disease Initiative Foundation for collecting patient data and samples in the ENROLL-HD platform.

Conflicts of Interest: All authors declare no conflict of interest. 


\section{References}

1. De Luca, A.; Morella, A.; Consoli, F.; Fanelli, S.; Thibert, J.R.; Statt, S.; Latham, G.J.; Squitieri, F. A Novel Triplet-Primed PCR Assay to Detect the Full Range of Trinucleotide CAG Repeats in the Huntingtin Gene (HTT). Int. J. Mol. Sci. 2021, 22, 1689. [CrossRef] [PubMed]

2. Migliore, S.; Jankovic, J.; Squitieri, F. Genetic Counseling in Huntington's Disease: Potential New Challenges on Horizon? Front. Neurol. 2019, 10, 453. [CrossRef] [PubMed]

3. Trinkler, I.; Cleret de Langavant, L.; Bachoud-Lévi, A.C. Joint recognition-expression impairment of facial emotions in Huntington's disease despite intact understanding of feelings. Cortex 2013, 49, 549-558. [CrossRef]

4. Glikmann-Johnston, Y.; Mercieca, E.C.; Carmichael, A.M.; Alexander, B.; Harding, I.H.; Stout, J.C. Hippocampal and striatal volumes correlate with spatial memory impairment in Huntington's disease. J. Neurosci. Res. 2021, 99, 2948-2963. [CrossRef]

5. Migliore, S.; D'Aurizio, G.; Curcio, G.; Squitieri, F. Task-switching abilities in pre-manifest Huntington's disease subjects. Parkinsonism Relat. Disord. 2019, 60, 111-117. [CrossRef]

6. Stern, Y. What is cognitive reserve? Theory and research application of the reserve concept. J. Int. Neuropsychol. Soc. 2002, 8 , 448-460. [CrossRef]

7. Stern, Y. Cognitive reserve. Neuropsychologia 2009, 47, 2015-2028. [CrossRef] [PubMed]

8. Barulli, D.; Stern, Y. Efficiency, capacity, compensation, maintenance, plasticity: Emerging concepts in cognitive reserve. Trends Cogn. Sci. 2013, 17, 502-509. [CrossRef]

9. McDowell, I.; Xi, G.; Lindsay, J.; Tierney, M. Mapping the connections between education and dementia. J. Clin. Exp. Neuropsychol. 2007, 29, 127-141. [CrossRef]

10. Staff, R.T.; Murray, A.D.; Deary, I.J.; Whalley, L.J. What provides cerebral reserve? Brain 2004, 127, 1191-1199. [CrossRef]

11. Solé-Padullés, C.; Bartrés-Faz, D.; Junqué, C.; Vendrell, P.; Rami, L.; Clemente, I.C.; Bosch, B.; Villar, A.; Bargalló, N.; Jurado, M.A.; et al. Brain structure and function related to cognitive reserve variables in normal aging, mild cognitive impairment and Alzheimer's disease. Neurobiol. Aging 2009, 30, 1114-1124. [CrossRef] [PubMed]

12. Stern, Y. Cognitive reserve in ageing and Alzheimer's disease. Lancet Neurol. 2012, 11, 1006-1012. [CrossRef]

13. Hindle, J.V.; Martyr, A.; Clare, L. Cognitive reserve in Parkinson's disease: A systematic review and meta-analysis. Parkinsonism Relat. Disord. 2014, 20, 1-7. [CrossRef] [PubMed]

14. Sumowski, J.F.; Leavitt, V.M. Cognitive reserve in multiple sclerosis. Mult. Scler. J. 2013, 19, 1122-1127. [CrossRef]

15. Elkins, J.S.; Longstreth, W.T., Jr.; Manolio, T.A.; Newman, A.B.; Bhadelia, R.A.; Johnston, S.C. Education and the cognitive decline associated with MRI-defined brain infarct. Neurology 2006, 67, 435-440. [CrossRef]

16. Kesler, S.R.; Adams, H.F.; Blasey, C.M.; Bigler, E.D. Premorbid intellectual functioning, education, and brain size in traumatic brain injury: An investigation of the cognitive reserve hypothesis. Appl. Neuropsychol. 2003, 10, 153-162. [CrossRef]

17. Dufouil, C.; Alpérovitch, A.; Tzourio, C. Influence of education on the relationship between white matter lesions and cognition. Neurology 2003, 60, 831-836. [CrossRef]

18. Andrews, S.C.; Domínguez, J.F.; Mercieca, E.C.; Georgiou-Karistianis, N.; Stout, J.C. Cognitive interventions to enhance neural compensation in Huntington's disease. Neurodegener. Dis. Manag. 2015, 5, 155-164. [CrossRef]

19. van Dellen, A.; Blakemore, C.; Deacon, R.; York, D.; Hannan, A.J. Delaying the onset of Huntington's in mice. Nature 2000, 404, 721-722. [CrossRef]

20. Curtin, P.C.; Farrar, A.M.; Oakeshott, S.; Sutphen, J.; Berger, J.; Mazzella, M.; Cox, K.; He, D.; Alosio, W.; Park, L.C.; et al. Cognitive Training at a Young Age Attenuates Deficits in the zQ175 Mouse Model of HD. Front. Behav. Neurosci. 2016, 9, 361. [CrossRef]

21. Pang, T.; Stam, N.C.; Nithianantharajah, J.; Howard, M.L.; Hannan, A.J. Differential effects of voluntary physical exercise on behavioral and brain-derived neurotrophic factor expression deficits in Huntington's disease transgenic mice. Neuroscience 2006, 141, 569-584. [CrossRef]

22. Bonner-Jackson, A.; Long, J.D.; Westervelt, H.; Tremont, G.; Aylward, E.; Paulsen, J.S.; PREDICT-HD Investigators and Coordinators of the Huntington Study Group (2013). Cognitive reserve and brain reserve in prodromal Huntington's disease. J. Int. Neuropsychol. Soc. 2013, 19, 739-750. [CrossRef]

23. Garcia-Gorro, C.; Garau-Rolandi, M.; Escrichs, A.; Rodriguez-Dechicha, N.; Vaquer, I.; Subira, S.; Calopa, M.; Martinez-Horta, S.; Perez-Perez, J.; Kulisevsky, J.; et al. An active cognitive lifestyle as a potential neuroprotective factor in Huntington's disease. Neuropsychologia 2019, 122, 116-124. [CrossRef] [PubMed]

24. Soloveva, M.V.; Jamadar, S.D.; Poudel, G.; Georgiou-Karistianis, N. A critical review of brain and cognitive reserve in Huntington's disease. Neurosci. Biobehav. Rev. 2018, 88, 155-169. [CrossRef] [PubMed]

25. Cain, K.K.; Flanigan, J.L.; Dalrymple, W.A.; Patrie, J.; Harrison, M.B.; Barrett, M.J. The Effect of Education on Symptom Onset and Severity of Huntington's Disease. Mov. Disord. Clin. Pract. 2021, 8, 555-562. [CrossRef] [PubMed]

26. López-Sendón, J.L.; Royuela, A.; Trigo, P.; Orth, M.; Lange, H.; Reilmann, R.; Keylock, J.; Rickards, H.; Piacentini, S.; Squitieri, F.; et al. European HD Network. What is the impact of education on Huntington's disease? Mov. Disord. 2011, 26, 1489-1495. [CrossRef]

27. Borroni, B.; Premi, E.; Bozzali, M.; Padovani, A. Reserve mechanisms in neurodegenerative diseases: From bench to bedside and back again. Curr. Med. Chem. 2012, 19, 6112-6118. [CrossRef]

28. Papoutsi, M.; Labuschagne, I.; Tabrizi, S.J.; Stout, J.C. The cognitive burden in Huntington's disease: Pathology, phenotype, and mechanisms of compensation. Mov. Disord. 2014, 29, 673-683. [CrossRef] 
29. Landwehrmeyer, G.B.; Fitzer-Attas, C.J.; Giuliano, J.D.; Gonçalves, N.; Anderson, K.E.; Cardoso, F.; Ferreira, J.J.; Mestre, T.A.; Stout, J.C.; Sampaio, C. Data Analytics from Enroll-HD, a Global Clinical Research Platform for Huntington's Disease. Mov. Disord. Clin. Pract. 2016, 4, 212-224. [CrossRef]

30. Kremer, H.P.H.; Hungtington Study Group. Unified Huntington's Disease Rating Scale: Reliability and consistency. Huntington Study Group. Mov. Disord. 1996, 11, 136-142. [CrossRef]

31. Marder, K.; Zhao, H.; Myers, R.H.; Cudkowicz, M.; Kayson, E.; Kieburtz, K.; Orme, C.; Paulsen, J.; Penney, J.B., Jr.; Siemers, E.; et al Rate of functional decline in Huntington's disease. Huntington Study Group. Neurology 2000, 54, 452-458. [CrossRef]

32. Schobel, S.A.; Palermo, G.; Auinger, P.; Long, J.D.; Ma, S.; Khwaja, O.S.; Trundell, D.; Cudkowicz, M.; Hersch, S.; Sampaio, C.; et al. TRACK-HD, COHORT, CARE-HD, and 2CARE Huntington Study Group Investigators. Motor, cognitive, and functional declines contribute to a single progressive factor in early HD. Neurology 2017, 89, 2495-2502. [CrossRef]

33. Nucci, M.; Mapelli, D.; Mondini, S. Cognitive Reserve Index questionnaire (CRIq): A new instrument for measuring cognitive reserve. Aging Clin. Exp. Res. 2012, 24, 218-226. [CrossRef]

34. Martínez-Horta, S.; Moreu, A.; Perez-Perez, J.; Sampedro, F.; Horta-Barba, A.; Pagonabarraga, J.; Gomez-Anson, B.; Lozano-Martinez, G.A.; Lopez-Mora, D.A.; Camacho, V.; et al. The impact of bilingualism on brain structure and function in Huntington's disease. Parkinsonism Relat. Disord. 2019, 60, 92-97. [CrossRef]

35. Nithianantharajah, J.; Hannan, A.J. Mechanisms mediating brain and cognitive reserve: Experience-dependent neuroprotection and functional compensation in animal models of neurodegenerative diseases. Prog. Neuro-Psychopharmacol. Biol. Psychiatry 2011, 35, 331-339. [CrossRef]

36. Cruickshank, T.; Bartlett, D.; Govus, A.; Hannan, A.; Teo, W.P.; Mason, S.; Lo, J.; Ziman, M. The relationship between lifestyle and serum neurofilament light protein in Huntington's disease. Brain Behav. 2020, 10, e01578. [CrossRef]

37. Kim, H.; Hong, J.P.; Kang, J.M.; Kim, W.H.; Maeng, S.; Cho, S.E.; Na, K.S.; Oh, S.H.; Park, J.W.; Cho, S.J.; et al. Cognitive reserve and the effects of virtual reality-based cognitive training on elderly individuals with mild cognitive impairment and normal cognition. Psychogeriatrics 2021, 21, 552-559. [CrossRef] [PubMed]

38. Lee, S.Y.; Kang, J.M.; Kim, D.J.; Woo, S.K.; Lee, J.Y.; Cho, S.J. Cognitive Reserve, Leisure Activity, and Neuropsychological Profile in the Early Stage of Cognitive Decline. Front. Aging Neurosci. 2020, 12, 590607. [CrossRef] [PubMed]

39. Tucker, A.M.; Stern, Y. Cognitive reserve in aging. Curr. Alzheimer Res. 2011, 8, 354-360. [CrossRef]

40. Bennett, D.A.; Wilson, R.S.; Schneider, J.A.; Evans, D.A.; Mendes de Leon, C.F.; Arnold, S.E.; Barnes, L.L.; Bienias, J.L. Education modifies the relation of AD pathology to level of cognitive function in older persons. Neurology 2003, 60, 1909-1915. [CrossRef] [PubMed]

41. Sumowski, J.F.; Rocca, M.A.; Leavitt, V.M.; Riccitelli, G.; Comi, G.; DeLuca, J.; Filippi, M. Brain reserve and cognitive reserve in multiple sclerosis: What you've got and how you use it. Neurology 2013, 80, 2186-2193. [CrossRef] [PubMed]

42. Neubauer, A.C.; Grabner, R.H.; Fink, A.; Neuper, C. Intelligence and neural efficiency: Further evidence of the influence of task content and sex on the brain-IQ relationship. Brain Res. Cogn. Brain Res. 2005, 25, 217-225. [CrossRef] [PubMed]

43. Cotman, C.W.; Berchtold, N.C. Exercise: A behavioral intervention to enhance brain health and plasticity. Trends Neurosci. 2002, 25, 295-301. [CrossRef] 